\title{
Comment on "Quantum-to-the-Home: Achieving Gbits/s Secure Key Rates via Commercial Off-the-Shelf Telecommunication Equipment"
}

\author{
Zhen Qu and Ivan B. Djordjevic $\mathbb{D}$ \\ Department of Electrical and Computer Engineering, University of Arizona, Tucson, AZ 85719, USA \\ Correspondence should be addressed to Ivan B. Djordjevic; ivan@email.arizona.edu \\ Received 3 November 2017; Accepted 12 June 2019; Published 18 July 2019 \\ Academic Editor: Frederik Armknecht
}

Copyright (c) 2019 Zhen Qu and Ivan B. Djordjevic. This is an open access article distributed under the Creative Commons Attribution License, which permits unrestricted use, distribution, and reproduction in any medium, provided the original work is properly cited.

The article titled "Quantum-to-the-Home: Achieving Gbits/s Secure Key Rates via Commercial Off-the-Shelf Telecommunication Equipment" [1] copied substantial parts of our previous publications without properly citing the source. In our previous publications we clearly described the mathematical model of our RF-subcarrier-assisted four-state continuousvariable QKD (CV-QKD) system, as well as the phase noise cancellation (PNC) stage $[2,3]$. While the article [1] copied our work without properly citing it, they cited one of our articles in an unremarkable position in Section 3 and put the core mathematical model and PNC model in the appendix, like their creative work. As an illustration, Figure 2 in their article can be found as Figure 1 of [2]. However, they attributed the PNC stage to their previous conference paper [4], which was presented in June 2017, well after the articles $[2,3]$ were published. Further, the derivations in Appendices $\mathrm{A}$ and $\mathrm{B}$ are copied from [2] with some small changes.

\section{Conflicts of Interest}

The authors declare that there are no conflicts of interest regarding the publication of this article.

\section{References}

[1] R. Asif and W. J. Buchanan, "Quantum-to-the-home: achieving gbits/s secure key rates via commercial off-the-shelf telecommunication equipment," Security and Communication Networks, vol. 2017, Article ID 7616847, 10 pages, 2017.
[2] Z. Qu, I. B. Djordjevic, and M. A. Neifeld, "RF-subcarrierassisted four-state continuous-variable QKD based on coherent detection," Optics Expresss, vol. 41, no. 23, pp. 5507-5510, 2016.

[3] Z. Qu and I. B. Djordjevic, "High-speed free-space optical continuous-variable quantum key distribution enabled by three-dimensional multiplexing," Optics Express, vol. 25, no. 7, pp. 7919-7928, 2017.

[4] R. Asif and W. J. Buchanan, "Seamless cryptographic key generation via off-the-shelf telecommunication components for end-to-end data encryption," in Proceeding of the 10th IEEE International Conference on Internet of Things (iThings)'17, June 2017. 


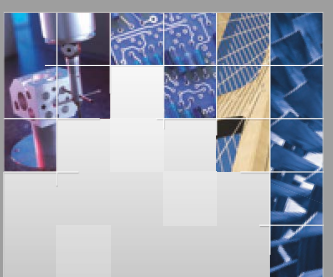

\section{Enfincering}
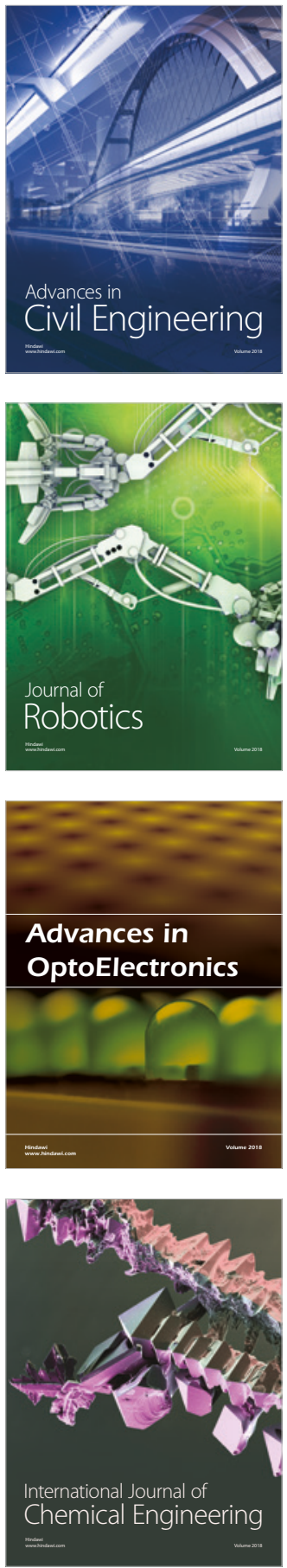

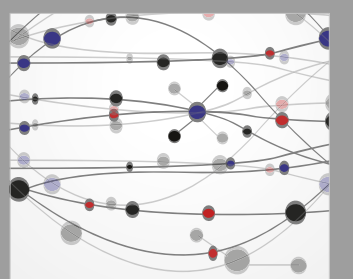

\section{Rotating \\ Machinery}

The Scientific World Journal

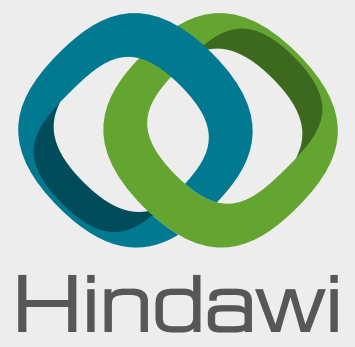

Submit your manuscripts at

www.hindawi.com
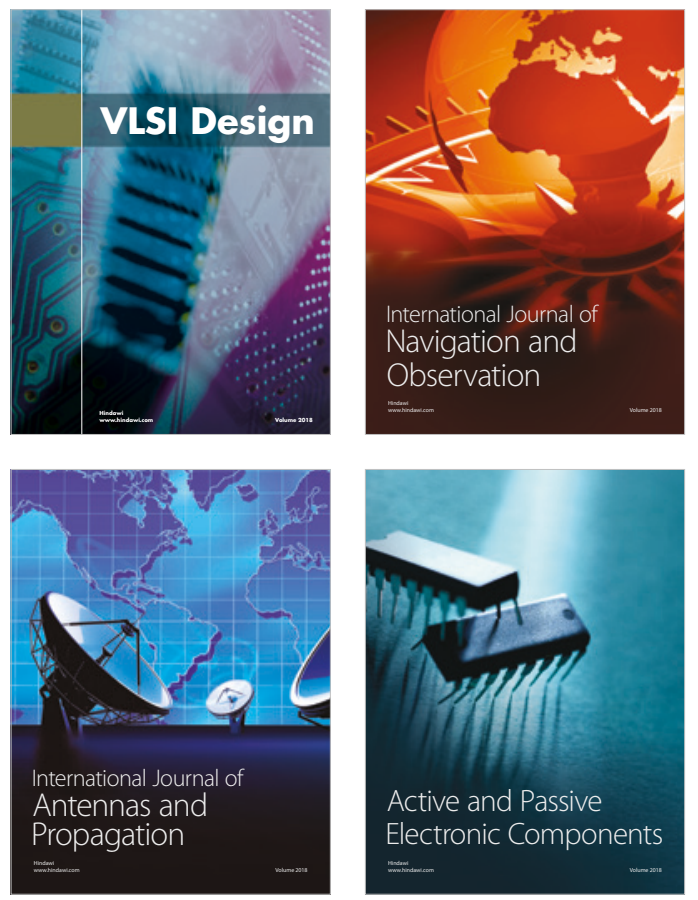
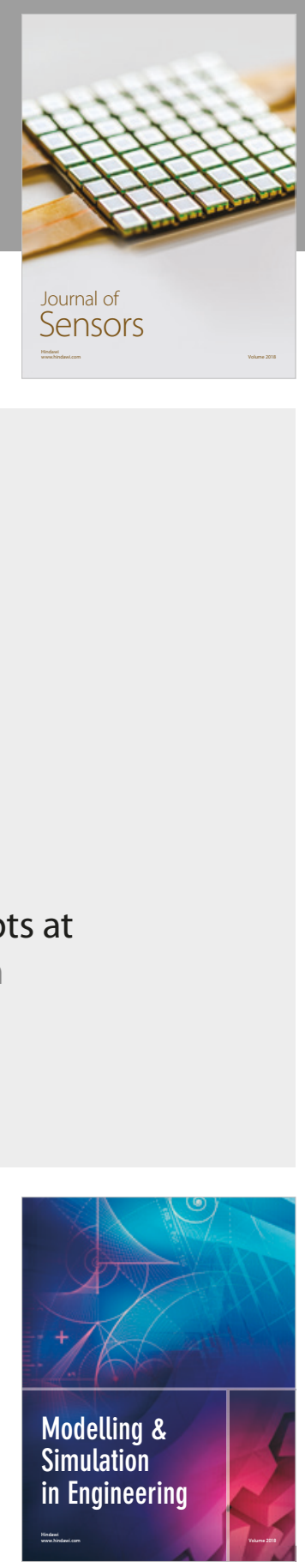

\section{Advances \\ Multimedia}
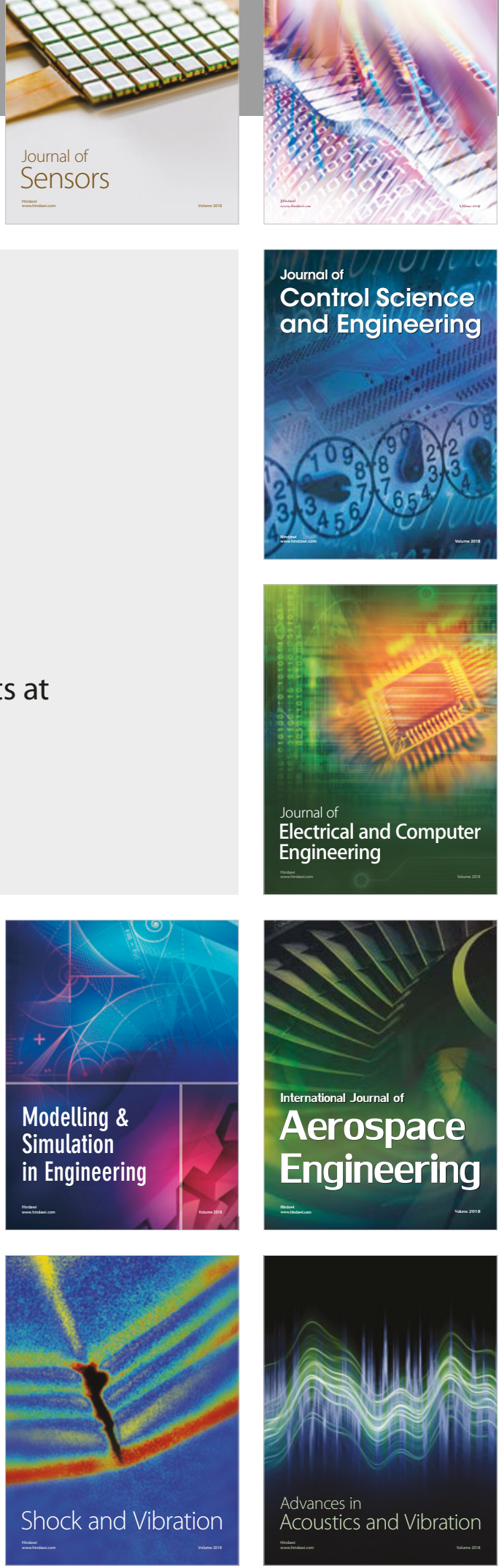